\title{
Subject Index Vol. 26, 2006
}

\section{Nephrology}

$\alpha_{1}$-Acid glycoprotein 59

Acute renal failure 388

Adiponectin 340

Adsorbent, oral 455

Advanced glycation end products 181

- _ _ products (AGE) 430

Albumin 67

Albuminuria 268, 621

Aldehydes 299

Aldosterone 194

Alkanals 299

Alkenals 299

ALT-711 (alagebrium) 430

Ambulatory blood pressure 462

Amino acid metabolism 310

Ammoniagenesis 194

Androgen deficiency 536

Anemia 149, 276, 571

Angiogenesis 97

Angiotensin 281

- I-converting enzyme gene 511

- II 170, 562, 590

Angiotensin-converting enzyme gene polymorphism 157

Anti-AGE therapy 430

Antidiuretic hormone 194, 348

Antioxidant 213

Apelin 121

Apolipoprotein 372

Apoptosis 400, 531

Aquaporins 579

Arginine vasopressin 579

Aristolochic acid 1

- - nephropathy 363

AST-120 136

Atherosclerosis 531

Atrial natriuretic peptide 590

Autosomal dominant polycystic kidney disease 335

Bacterial cultures, peritoneal dialysis patients 115

- RNA amplification 115

Balkan endemic nephropathy, etiology 1

- $\quad$-, genetic predisposition 1
Bicarbonate secretion 194

Blood flow 335

- pressure 503

Booster vaccination, influenza 206

$\mathrm{BSC}_{1} 245$

Candesartan, pleiotropic effects 462

Cardiac function 121

Cardiovascular disease(s) 59, 503

- mortality 408

- remodeling 340

CC-chemokines 497

CD40 356, 602

Chemokine 319

Childhood nephrosis 258

Children, steroid resistance 544

Cholesterol 67

Chronic allograft nephropathy 319

- kidney disease(s) 59, 136, $142,149,408,423,445,483$, 503

- - diseases 483

- renal failure 276, 437

- $\quad$ injury 97

c-Jun N-terminal kinase 281

c-met 519

Connective tissue growth factor 400

Continuous ambulatory peritoneal dialysis peritonitis 115

Contrast nephropathy 287

Coronary artery disease 121

C-reactive protein 59,91

Creatinine clearance 612

Cyclosporine 163, 400

Cytokines, urinary 483

Darbepoetin alfa 149,571

Dermatan sulphate 621

Diabetes 163

- mellitus 87, 340

Diabetic nephropathy 50, 415, $430,476,562,621$

Dialysis access 181

- membrane 372

Dietary glycotoxins 181

- sodium 268
Dilatory responses 335

Doppler ultrasound 16

Dose conversion 571

Enalapril 281, 562

End stage renal disease 469

Endothelial dysfunction 335 , 536

- NOS 245

Endothelin-B receptors 22

End-stage kidney disease 536

- renal disease 142, 340, 408

Enzyme immunoassay 186

Epithelial-to-mesenchymal transition 381

Epoetin alfa 571

Erythropoiesis 149

Erythropoietin 276, 326

E-selectin 437

Essential hypertension 16

Estrogen 170

Factor H-deficient mice 127

Familiality, chronic kidney disease/end-stage renal disease 142

Fetuin-A 423

Fibrosis 50

Five-sixths renal ablation 281

Free iron 304

G-308A polymorphism 12

Gender differences, high-molecular-weight adiponectin 476

Gene therapy 326

Genetic polymorphism 12

Genetics 218

-, kidney diseases 142

Glomerular filtration rate 268 , 408

- injury 186

Glomerulonephritis 75

Glucose 163

Graft thrombosis 181

Heme oxygenase 552

Hemin 552

Hemodialysis $121,299,423$, 437, 469, 497, 536, 571

- patients influenza vaccination 206

Hemodynamics 388
Heparin 621

Hepatocyte growth factor 519

High-molecular-weight adiponectin 476

Histopathological changes 491

HMG-CoA reductase inhibitor 82

4-HO-alkenals 299

Homeostasis 348

Hospital 571

Hypercalcemia 105

Hyperinsulinemia 232

Hyperphosphatemia 105

Hypertension 170, 388, 415, 552

-, essential 503

Hyponatremia 348

-, vasopressin dysregulation 579

Hypoxia 22

Hypoxia-inducible factor 22

ICAM-1 602

ICAT labeling 127

Immunoglobulin A nephropathy 43,511

Inflammation 181, 356, 415, 531

Influenza, vaccination 206

Inner medullary collecting duct 194

Insertion/deletion 511

Insulin 232

- resistance 232,245

Interleukin 1562

- 6562

Interleukin-6 75, 91

Intermittent dosing, paricalcitol 105

Interstitial fibrosis 34

Intimal hyperplasia 181

Intima-media thickness 462, 497

Intravenous iron 304

Iron supplementation, intravenous versus oral 445

Ischemia/reperfusion 213

Ischemia-reperfusion 22

-, urinary nitrate/nitrite 388

Isoprenylation 381 
Ketoconazole-tacrolimus kidney transplant 293

Kidney 67, 163, 232

- disease 268

- failure, acute 22

- -, chronic 22

- regeneration 34

- transplant 213

- transplantation 319,491

Left ventricle filling diseases 469

Lentiviral vector 326

Live donors 491

Liver 310

Liver-type fatty-acid-binding protein 82,276

Losartan 50

Lower extremity revascularization 612

Macrophage 415

Medulla 22

Membranoproliferative glomerulonephritis 127

Membranous glomerulonephritis 12

Mesangial cells 519

Microalbuminuria 462

Microdialysis 163

Molecular biology 218

Monocyte chemoattractant protein-1 319

MUC20 gene 43

Myocardial infarction 408

Nephrotic syndrome 157, 258, 544

Nephrotoxicity 163, 400

Neutrophil-gelatinaseassociated lipocalin 287

Nitric oxide 170, 213, 310

Nuclear factor-кB 281

Ochratoxin A 1

Once-daily dosing, paricalcitol 105

Oral adsorbent 136

Outflow dialysate 372
Oxidative injury 552

- stress 415, 455, 519

p38 mitogen-activated kinase 319

Partial nephrectomy 326

Pentoxifylline 562

Percutaneous coronary intervention 287

Peripheral blood mononuclear cells 91

Peritoneal cavity local defense 602

- dialysis 115

- equilibration test 87

- mesothelial cells, rat 602

Peritubular capillary loss 363

Peroxisome proliferator activated receptor gamma 67

- proliferator-activated receptor- $\gamma$ 356, 602

Pimonidazole 22

Pioglitazone 476

Placenta growth factor 97

Plasma L-arginine 310

Polycyclic aromatic hydrocarbons 1

Polymerase chain reaction, infectious disease detection 115

Polymorphism 511

Preload increase maneuver 469

Protein 372

- array analysis 483

- metabolism 310

- modification 299

Proteinase inhibitor 834

Proteinuria 67, 82, 186, 268, 430

Proteome 258, 372

Proteomic profiling 127

Protocol biopsy 491

Proximal tubule 82

P-selectin 437

Pulse dosing, paricalcitol 105

- wave velocity 462
Rac1 activation 381

Radiologic contrast media 22

RANTES 356

Reactive oxygen species 519

Recombinant human erythropoietin 497

Renal cancer 218

- fibrosis 281, 381

- function 136, 335

- -, diabetes 430

- injury 232

- resistive index 16

- tubular epithelial cells 356

RhoA activation 381

Salt intake 268

- sensitivity 552

Salt-water balance 348

SELDI 258

SELDI-TOF-MS 372

Serpinb8 34

Serum albumin 91

- creatinine 136,612

sICAM-1 437

Sirolimus 400

Small RhoGTPase 381

Sodium 170, 348

- overload 590

Soluble gp130 91

- interleukin-6 receptor 91

Spontaneously hypertensive rats 415

Staphylococcus aureus infection 304

Statins 381

Steroid dependence 157

- sensitivity 258

Steroid-resistant FSGS, children 544

- -, management 544

Streptococcal erythrogenic toxin 75

Subcutaneous fat area 476

Sulodexide 621

sVCAM-1 437

Syndrome of inappropriate secretion of antidiuretic hormone 348
Tacrolimus treatment in children 544

Telomeres 91

Thiazolidinediones 67

Tissue Doppler imaging 469

TNFp55 437

TNFp75 437

Transforming growth factor- $\beta_{1}$ 186

Transplantation 287

Tubular dysfunction 621

Tubulointerstitial fibrosis 363

- injury 552

- nephropathy 16

Tumor necrosis factor- $\alpha 12$, 562

Type 2 diabetes 245

_ _ _ mellitus 476

Ultrafiltration 87

Unilateral ureteral obstruction 34

Urea production 310

Uremic rats 326

- toxin 455

Uric acid 388

Urinary albumin 50

- biomarkers 258

Urine protein profiling 127

Uromodulin 127

Urothelial cancer 1

Utah population data base (UPDB) 142

Variable number of tandem repeats polymorphism 43

Vascular access 304

- calcification 423

- smooth muscle cell 531

Vasopressin 348

Vasopressin-receptor antagonist, SIADH 579

Visceral fat area 476

Vitamin D 105

- D receptor activator 105

Xenobiotic-metabolizing genes 1 